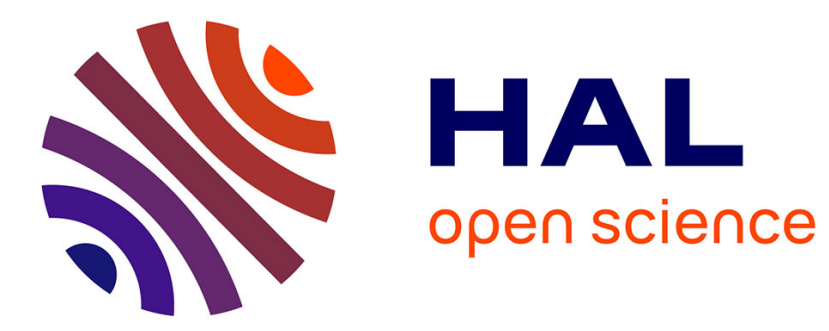

\title{
Instability of a fluid inside a precessing cylinder
}

Romain Lagrange, Christophe Eloy, François Nadal, Patrice Meunier

\section{To cite this version:}

Romain Lagrange, Christophe Eloy, François Nadal, Patrice Meunier. Instability of a fluid inside a precessing cylinder. Physics of Fluids, 2008, 20 (8), pp.081701. 10.1063/1.2963969 . hal-03347583

\section{HAL Id: hal-03347583 \\ https://hal.science/hal-03347583}

Submitted on 17 Sep 2021

HAL is a multi-disciplinary open access archive for the deposit and dissemination of scientific research documents, whether they are published or not. The documents may come from teaching and research institutions in France or abroad, or from public or private research centers.
L'archive ouverte pluridisciplinaire HAL, est destinée au dépôt et à la diffusion de documents scientifiques de niveau recherche, publiés ou non, émanant des établissements d'enseignement et de recherche français ou étrangers, des laboratoires publics ou privés. 


\title{
Instability of a fluid inside a precessing cylinder
}

\author{
Romain Lagrange*, Christophe Eloy*, François Nadal ${ }^{\dagger}$ and Patrice Meunier* \\ *IRPHE, CNRS \& Aix-Marseille Université, \\ 49 rue Joliot-Curie, 13013 Marseille, France \\ $\dagger$ Commissariat à l'Énergie Atomique \\ CESTA 33114 Le Barp, France
}

\begin{abstract}
In this paper, we report experimental results on the stability of a fluid inside a precessing and resonant cylinder. Above a critical Reynolds number, the Kelvin mode forced by precession triggers an instability which saturates at intermediate Re and which leads to a turbulent flow at high Reynolds numbers. Particle Image Velocimetry measurements in two different sections of the cylinder have revealed the 3D structure of this instability. It is composed of two free Kelvin modes whose wavenumbers and frequencies respect the conditions for a triadic resonance with the forced Kelvin mode, as is obtained for the elliptical instability. Moreover, an experimental diagram of stability has been established by varying both the precessing angle and the Reynolds number. It shows a good agreement with a scaling analysis based on a triadic resonance mechanism.
\end{abstract}

The knowledge of the flow forced by a precessional motion is of critical importance in several domains. In aeronautics, the liquid propellant contained in a flying object can be forced by precession. The resulting flow can create a destabilizing torque on the object and thus modify its trajectory dangerously. In geophysics the Earth's precession modifies the flow of its liquid core and is therefore of signicative importance in understanding the geodynamo (among other effects such as convection, boundary layers, elliptic or tidal instability [1]). The flow inside a cylinder subjected to precession can be decomposed as a sum of a shear along the cylinder axis and a superposition of Kelvin modes which become resonant for particular precession frequencies. McEwan [2] first observed that this flow can become unstable and even turbulent for large Reynolds numbers. This behavior has also been reported by Manasseh [3-5], and Kobine [6]. Several scenarios have been proposed to explain this instability. Studying the case of an infinite cylinder, Mahalov [7] proposed a mechanism of triadic resonance between the flow shear and two Kelvin modes. Kerswell [8] suggested that a given Kelvin mode can trigger a triadic resonance with two other Kelvin modes leading to an instability. Another scenario, suggested by Kobine $[6,9]$ is that the main flow could be modified by a geostrophic mode (due to nonlinear effects) eventually leading to a centrifugal instability.

An experimental set-up has been built to study the precession of a cylinder of height $H$ along its axis $\hat{\mathbf{z}}$ and radius $R$, full of water of kinematic viscosity $\nu$. More details about the set-up can be found in [10]. The cylinder rotates at the angular frequency $\Omega_{1}$ (measured with an accuracy of $0.1 \%$ ) around its axis. It is mounted on a platform which rotates at the angular frequency $\Omega_{2}$ (measured with an accuracy of $0.2 \%$ ). Once the spin-up stage is completed, the cylinder is tilted with an angle $\alpha$

\footnotetext{
*lagrange@irphe.univ-mrs.fr
}

(determined with an absolute accuracy of $\pm 0.1^{\circ}$ ) with respect to the rotation axis of the platform. Particle Image Velocimetry (PIV) measurements in transverse sections of the cylinder are made. To perform the acquisition of a PIV field, we use small markers illuminated with a thin light sheet created by a Yag pulsed laser. The particle images are recorded by a camera mounted on the rotating platform. The horizontal velocity and the axial vorticity fields in the cylinder frame of reference are thus measured. More details about PIV treatment can be found in [11].

In the following, variables are made dimensionless by using $R$ and $\Omega=\Omega_{1}+\Omega_{2} \cos \alpha$ as characteristic length and characteristic frequency. The dynamics of this precessing system depends on four dimensionless numbers: the aspect ratio $h=H / R$, the frequency ratio $\omega=\Omega_{1} / \Omega$, the Rossby number Ro $=\Omega_{2} \sin \alpha / \Omega$ and the Reynolds number $\operatorname{Re}=\Omega R^{2} / \nu$. The cylindrical coordinates are used in the reference frame of the cylinder and noted $(r, \theta, z)$, where $z=0$ corresponds to the mid-height section of the cylinder.

Figure 1 shows the axial and instantaneous flow vorticity for a small precessing angle $\left(\alpha=1^{\circ}\right)$ and different Reynolds numbers. The laser sheet is at an altitude $z \simeq h / 4$. For $\operatorname{Re}=3500$ (Fig. 1a), the flow mainly consists of two stationary counter rotating vortices. A classical linear and inviscid theory is sufficient to explain this observation. By assuming a small Rossby number (weak precession, negligible nonlinear effects) and a large Reynolds number (negligible viscous effects), the linearized Euler equation at order $O(\mathrm{Ro})$ is

$$
\frac{\partial \mathbf{v}}{\partial t}+2 \hat{\mathbf{z}} \times \mathbf{v}+\nabla p=-2 \operatorname{Ro} \omega r \cos (\omega t+\theta) \hat{\mathbf{z}},
$$

where $2 \hat{\mathbf{z}} \times \mathbf{v}$ is the dimensionless Coriolis force and $p$ the dimensionless pressure including all potential terms. The right-hand side of Eq. (1) is the precession forcing which forces a particular solution of Eq. (1): $\mathbf{v}_{\text {part }}=$ $-2 \operatorname{Ro} r \sin (\omega t+\theta) \hat{\mathbf{z}}$. This solution does not satisfy the boundary conditions of no outward flow at $z= \pm h / 2$. 
(a)

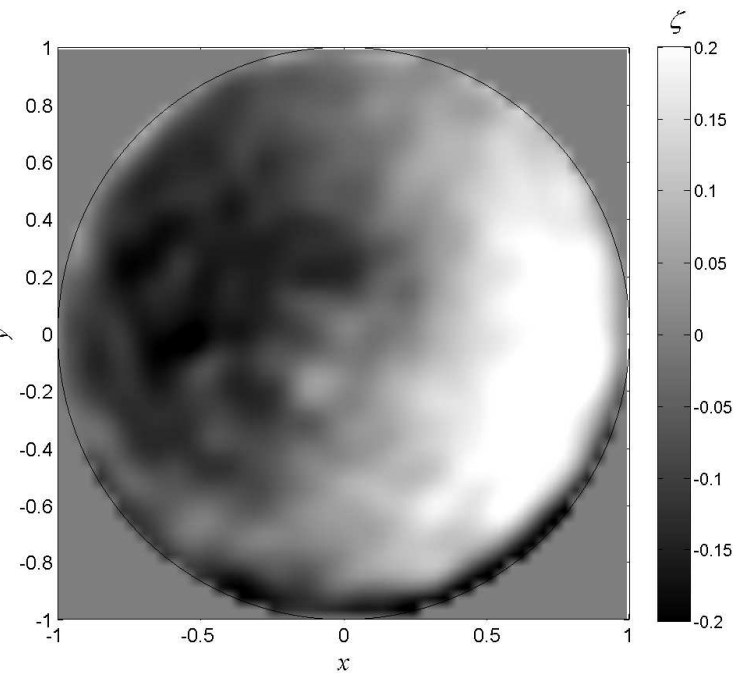

(b)
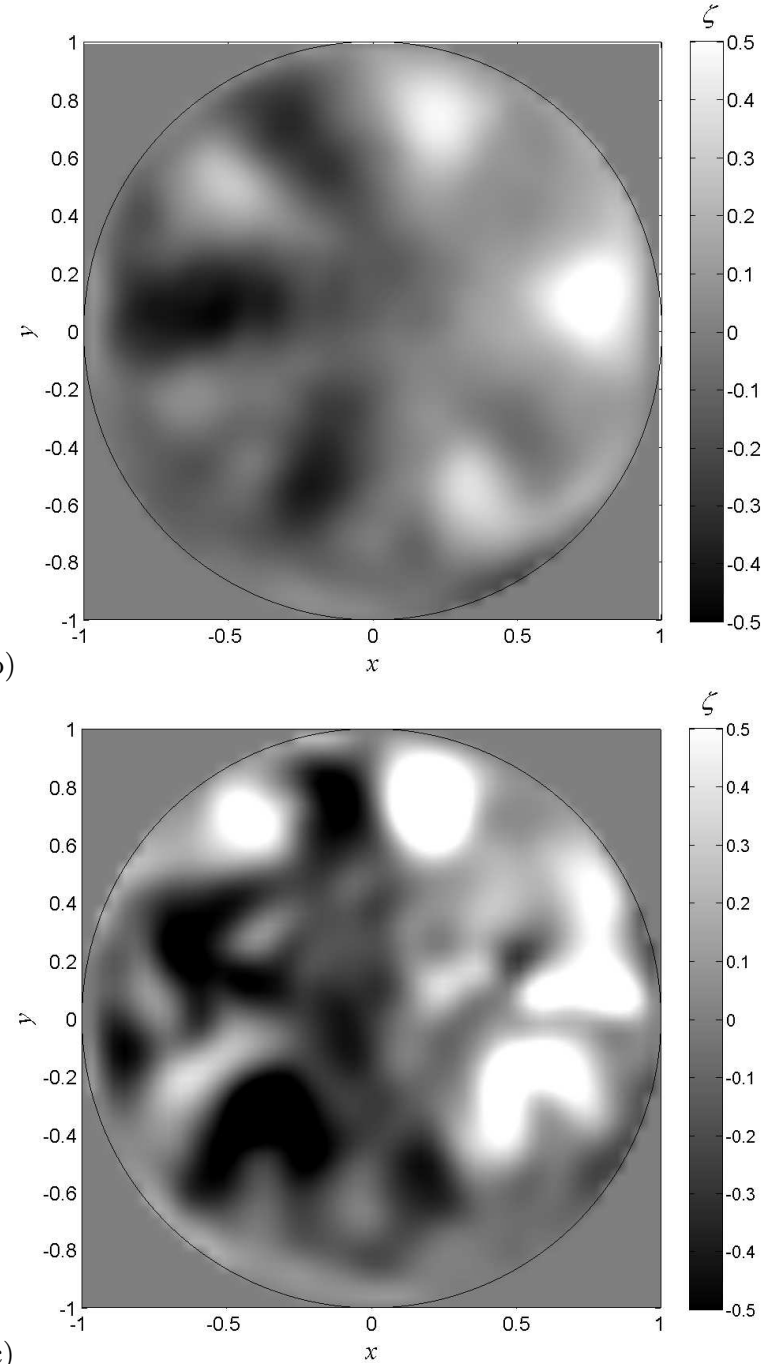

FIG. 1: Axial vorticity $\zeta$ of the flow for different Reynolds numbers at $z=h / 4$. (a) For $\operatorname{Re}=3500$ the stable flow exhibits the forced Kelvin mode. (b) For Re $=6000$ the flow is unstable and exhibits a free Kelvin mode with $m_{1}=5$ superimposed to the forced Kelvin mode. The temporal evolution of the instability can be observed in the corresponding movie. (c) For $\operatorname{Re}=24400$ the flow is turbulent. For these three cases $h=1.62, \omega=1.18, \mathrm{Ro}=0.0031$.
Thus, we must complete this solution with a solution of the homogeneous equation (Eq. (1) without forcing), so that the boundary condition at the upper and lower walls is satisfied. Due to time and azimuthal dependance of the forcing, the homogeneous solution is a sum of Kelvin modes of azimuthal wavenumber $m=1$ and angular frequency $\omega$. Finally the solution of Eq. (1) is

$$
\mathbf{v}=\mathbf{v}_{\text {part }}+\sum_{i=1}^{\infty} a_{i} \mathbf{v}_{i}\left(m=1, \omega, k_{i}\right),
$$

where $\mathbf{v}_{i}\left(m, \omega, k_{i}\right)$ is a Kelvin mode of amplitude $a_{i}$ and whose axial wavenumber $k_{i}$ depends on $\omega$ by the dispersion relation

$$
\begin{aligned}
\omega k_{i} \sqrt{4 / \omega^{2}-1} J_{m}^{\prime}\left(k_{i} \sqrt{4 / \omega^{2}-1}\right) \\
+2 J_{m}\left(k_{i} \sqrt{4 / \omega^{2}-1}\right)=0
\end{aligned}
$$

where $J_{m}$ is the Bessel function of the first kind, $J_{m}^{\prime}$ its derivative. The axial vorticity $\zeta_{i}$ of the $i$-th Kelvin mode is

$$
\zeta_{i}=J_{m}\left(k_{i} \sqrt{4 / \omega^{2}-1} r\right) \sin \left(k_{i} z\right) \cos (\omega t+m \theta) .
$$

When $k_{i}$ is equal to $(2 n+1) \pi / h$, with $\mathrm{n}$ an integer number, the $i$-th Kelvin mode 'fits' inside the height of the cylinder and becomes resonant. In our experiments (i.e for $h=1.62$ and $\omega=1.18$ ) the first Kelvin mode (which is theoretically characterized by two lobes of vorticity) is resonant (its axial wavenumber, noted $k$, is equal to $\pi / h$ ). Because the amplitude $a_{1}$ is predicted to diverge by a linear analysis it is necessary to include viscous [12] and nonlinear effects. We have shown in [10] that $a_{1}$ scales as $\operatorname{RoRe}^{1 / 2}$ for low Reynolds numbers (viscous regime, $\left.\mathrm{Re}^{1 / 2} \mathrm{Ro}^{2 / 3} \ll 1\right)$ and as $\mathrm{Ro}^{1 / 3}$ for large Reynolds numbers (nonlinear regime, $\operatorname{Re}^{1 / 2} \operatorname{Ro}^{2 / 3} \gg 1$ ). Since the nonresonant mode amplitudes scale as Ro, the resonant mode is always predominant.

Figure $1 \mathrm{~b}$ is a PIV measurement of the axial and instantaneous vorticity field for $\mathrm{Re}=6500$. For such a value of Re the flow seen in Fig. 1 is unstable and the unstable mode exhibits a ring with 10 lobes of vorticity with alternate sign. It corresponds to a free Kelvin mode (i.e a solution of Eq. (1) without forcing) whose azimuthal wavenumber, noted $m_{1}$ equals 5 . This mode $m_{1}=5$ is superimposed to the forced Kelvin mode $m=1$ shown in Fig. 1a. (As seen on Fig. 1b the average vorticity is negative for $x<0$ and positive for $x>0$ ). Such a flow, which is three-dimensional and non-stationary, corresponds to the instability discovered by McEwan [2] and studied by Manasseh [3] using visualizations, which was called "resonant collapse" since it decreases the amplitude of the forced Kelvin mode. Indeed, the same structure has been observed for other aspect ratios $(h=1.8$ and $h=2)$ and it also leads to the decrease of the forced Kelvin mode's amplitude. The visualization of a sequence of instantaneous PIV fields shows that the free Kelvin mode rotates as a function of time at a dimensionless frequency 


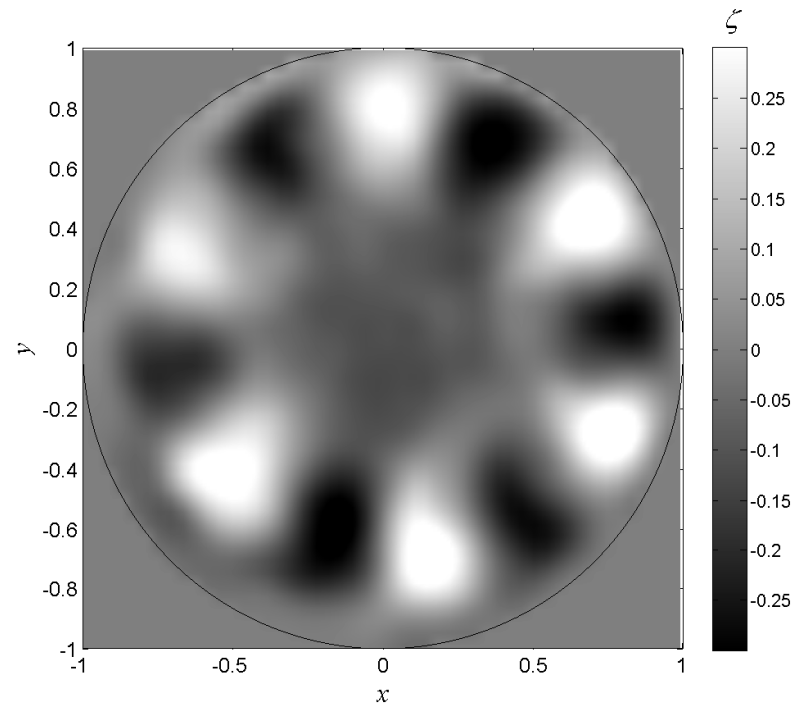

FIG. 2: Vorticity field of the unstable flow at mid-height of the cylinder for the same parameters as in figure $1(\mathrm{~b})(h=1.62$, $\omega=1.18, \operatorname{Ro}=0.0031, \operatorname{Re}=6500)$.

$\omega_{1}=-0.34 \pm 11 \%$ in the cylinder frame of reference. For this Reynolds number, the unstable mode beats probably due to a nonlinear coupling with the geostrophic mode. However, the amplitude of this unstable mode is stationary close to the threshold (i.e. $\mathrm{Re} \simeq 4600$ ).

Figure 1c represents the axial and instantaneous vorticity field for even larger Reynolds numbers ( $\mathrm{Re}=$ 24400). For such a value of Re the flow is disordered and seems to be turbulent. As suggested by Kerswell [8], this disordered flow could be the result of successive instabilities: a cascade of bifurcations could lead to a turbulent state. It can be noted that the Kelvin mode $m=1$ forced by precession is still present since the average vorticity is still negative for $x<0$ and positive for $x>0$.

Figure 2 is a PIV measurement of the axial and instantaneous vorticity field measured in a section of the cylinder lower than in Fig. 1. The laser sheet is at midheight of the cylinder $(z=0)$. According to Eq. (4) the vorticity of the forced Kelvin mode $m=1$ and of the free Kelvin mode $m_{1}=5$ is equal to 0 . At this altitude a structure with 12 lobes of alternate vorticity is clearly observed. It corresponds to a free Kelvin mode whose azimuthal wavenumber, noted $m_{2}$, is equal to 6 . Because it does not vanish at $z=0$ its axial vorticity is given by Eq. (4) where $\sin \left(k_{2} z\right)$ has been changed in $\cos \left(k_{2} z\right)$. This free Kelvin mode rotates at a dimensionless angular frequency $\omega_{2}=0.79 \pm 2.5 \%$ in the cylinder frame of reference.

The axial velocity (which is in quadrature with respect to the axial vorticity given by Eq. (4)) of the free Kelvin mode $m_{1}=5$ (resp. $\left.m_{2}=6\right)$ is a cosine (resp. sine) function of $z$. Boundary conditions of no outward flow at $z= \pm h / 2$ imply that the axial wavenumber of the free Kelvin mode $m_{1}=5$ (resp. $m_{2}=6$ ) is discretized

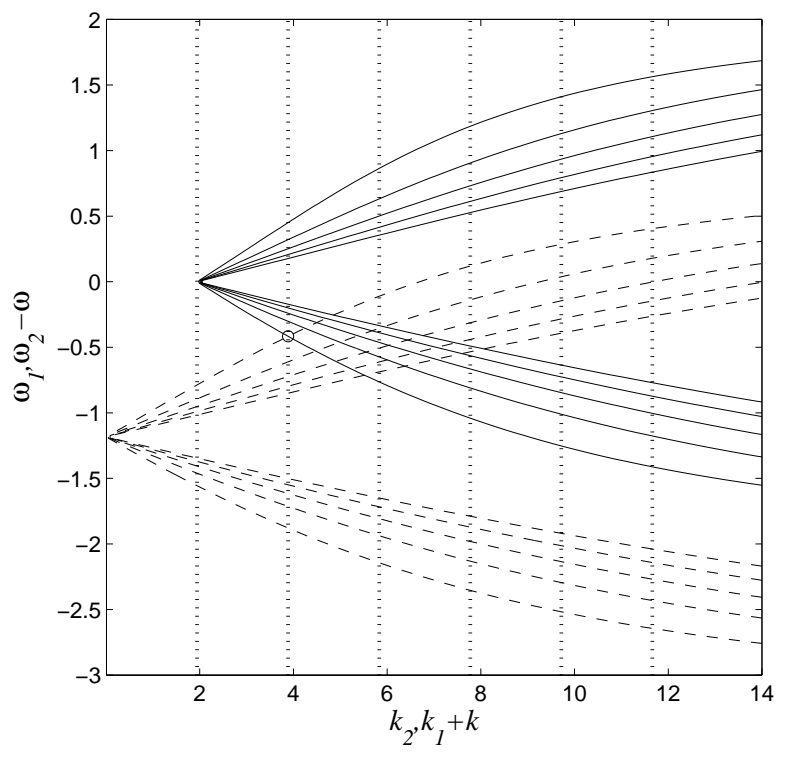

FIG. 3: Dispersion relations of the Kelvin modes. The solid lines (resp. dashed lines) correspond to the first five branches of the Kelvin modes with azimuthal wavenumber $m_{1}=5$ (resp. $m_{2}=6$ ). Solid lines have been translated by $k=\pi / h$ and dashed lines have been translated by $\omega=1.18$. Vertical dotted lines correspond $k=n \pi / h$, with $n$ an integer, $(h=$ $1.62, \omega=1.18)$.

as follows: $k_{1}=\left(2 n_{1}+1\right) \pi / h\left(\right.$ resp. $\left.k_{2}=2 n_{2} \pi / h\right), n_{1}$ (resp. $n_{2}$ ) being an integer.

Furthermore, figure $1 \mathrm{~b}$ and figure 2 show that the unstable Kelvin modes correspond to the first branch of the dispersion relation since there is only one ring of vortices. We can thus infer that $k_{1}=\pi / h$ (resp. $\left.k_{2}=2 \pi / h\right)$ since the point $\left(k_{1}=\pi / h, \omega_{1} \approx-0.34\right)$ (resp. $\left.\left(k_{2}=2 \pi / h, \omega_{1} \approx 0.79\right)\right)$ then falls very close to the first branch of the dispersion relation (3) for $m_{1}=5$ (resp. $m_{2}=6$ ) (Fig. 3).

These experiments have allowed to determine the structure of the instability of a fluid inside a precessing and resonant cylinder. We have found that the unstable flow is the sum of three Kelvin modes: the forced one, and two free modes. The azimuthal wavenumber and the angular frequency of these free modes have been measured and satisfy the conditions for a triadic resonance with the forced Kelvin mode

$$
m_{2}-m_{1}=1, \omega_{2}-\omega_{1} \approx \omega, k_{2}-k_{1}=k,
$$

where $k=\pi / h$ is the axial wavenumber of the forced Kelvin mode. This suggests that the nonlinear coupling of the three Kelvin modes can trigger an instability, in a similar way as for the elliptical instability [13, 14].

The resonant condition given in Eq. (5) corresponds to the crossing points of the dashed and solid lines in Fig. 3, where the two dispersion relations are plotted in the same plane; the dispersion relation with $m_{1}=5$ (resp. $m_{2}=$ 


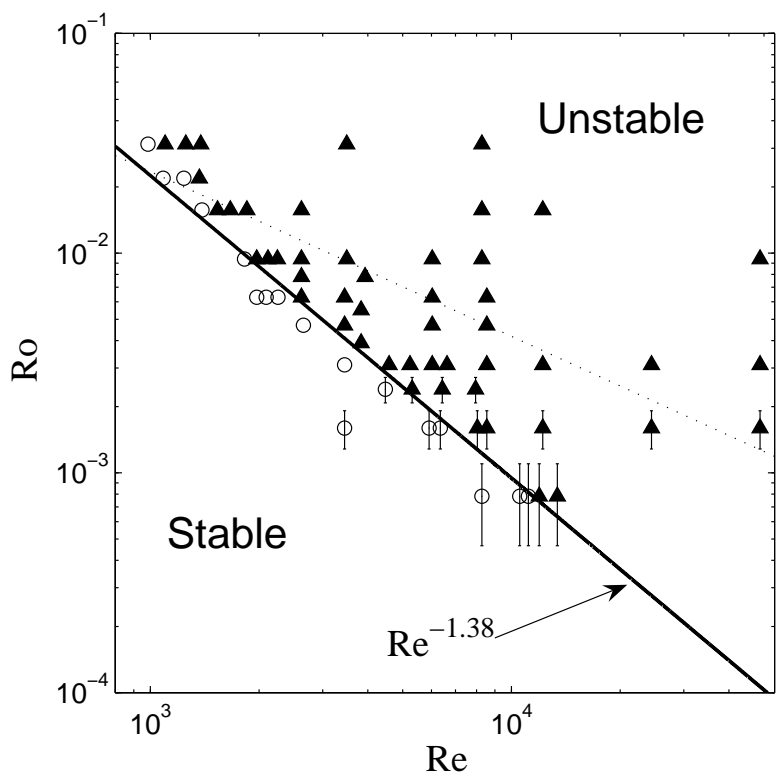

FIG. 4: Stability diagram of the flow inside a precessing cylinder for $(h=1.62, \omega=1.18)$. Circles represent stable experiments. Blacks triangles represent unstable experiments. The solid line is an experimental 'fit' of the threshold. The dashed line separates the viscous and the nonlinear domains of the base flow.

6) being horizontally (resp. vertically) translated of $k$ (resp. translated of $-\omega$ ). It can be noted that there is an infinite and denumerable number of possible resonances. However, the free Kelvin modes observed experimentally correspond to the crossing point surrounded by a circle on Fig. 3. These modes satisfy exactly the boundary conditions at $z= \pm h / 2$ (i.e. the crossing point lies on a vertical dotted line in Fig. 3). This exact resonance is only valid for $h=1.62$. For $h \approx 1.62$ 'detuning' effects shall come into play and thus decrease the instability growth rate.

For $h \simeq 1.62$ two free Kelvin modes involving different branches of the dispersion relations or different azimuthal wavenumbers $m_{1}$ and $m_{2}$ can exactly resonate with the forced Kelvin mode. Nevertheless, it can be shown that there can not be exact resonances for $m_{1} \leq 4$ for the first branches of the dispersion relations. Thus, the aspect ratio $h=1.62$ corresponds to the exact resonance of the Kelvin modes with the smallest wavenumbers. Since volume viscous effects increase with the wavenumbers of the free Kelvin modes, $h=1.62$ is expected to be the aspect ratio for which the flow is the most unstable. However, the previous observations are very general and do not depend on the fact that the triadic resonance is exact or not. Indeed, experiments with an arbitrary aspect ratio
( $h=1.8$ ) have shown exactly the same instability.

Finally we have plotted in Fig. 4 the stability diagram of this instability in the Re-Ro plane. The majority of the experiments close to threshold are in the viscous domain for the base flow. This means that the amplitude of the forced mode scales as $a_{1} \sim \operatorname{RoRe}^{1 / 2}$ [10]. Based on similarities with the elliptic instability, the inviscid growth rate $\sigma$ of the present triadic instability is expected to scale as the amplitude of the forced Kelvin mode: $\sigma \sim a_{1}$. The natural decay rate of Kelvin modes is due both to the boundary viscous layers and volume viscous effects. The surface (resp. volume) decay rate $\sigma_{\text {surf }}$ (resp. $\sigma_{\text {vol }}$ ) scales as $\sigma_{\text {surf }} \sim-\operatorname{Re}^{-1 / 2}$ (resp. $\left.\sigma_{\mathrm{vol}} \simeq-\left[m_{1}+k_{1}^{2}\left(4 / \omega^{2}-1\right)\right] \operatorname{Re}^{-1}\right)$. In our experiments, $\sigma_{\text {surf }} \simeq \sigma_{\text {vol }}$ for $\operatorname{Re} \simeq 3000$. When the instability is saturated by volume (i.e. Re $<3000$ ), (resp. boundary, i.e. $\operatorname{Re}>3000)$ viscous effects, the amplitude of the forced Kelvin at which the flow becomes unstable satisfies $a_{1 \mathrm{c}} \sim \operatorname{Re}^{-1}$ (resp. $a_{1 \mathrm{c}} \sim \mathrm{Re}^{-1 / 2}$ ). Thus the Rossby number at which the flow becomes unstable scales as $\mathrm{Ro}_{\mathrm{c}} \sim \mathrm{Re}^{-3 / 2}$ (resp. $\mathrm{Ro}_{\mathrm{c}} \sim \mathrm{Re}^{-1}$ ). A 'fit' of the experimental threshold gives $\operatorname{Ro}_{\mathrm{c}} \sim \mathrm{Re}^{-1.38}$ (solid line), which is coherent with the theoretical scalings.

In this paper we studied experimentally the flow inside a precessing and resonant cylinder. At a given Rossby number the flow is stable for small enough Reynolds numbers and exhibits a Kelvin mode forced by the precessional motion. Increasing the Reynolds number above a critical value the flow becomes unstable (and even turbulent for high Re). Measurements in two different cylinder sections have revealed the presence of two Kelvin modes with high azimuthal wavenumbers. Their frequencies and their wavenumbers satisfy the conditions for a triadic resonance with the forced Kelvin mode. Thus, this paper has confirmed the scenario suggested by Kerswell [8] that a Kelvin mode can be destabilized by a triadic resonance mechanism. So, the precessional instability is very general since it appears as soon as a Kelvin wave has been excited (through precession, compression, in the nonlinear stages of the elliptical instability or in the turbulent flow of a rotating cylinder). A stability diagram has also been established and showed that the scaling of the critical Ro as a function of Re is coherent with standard scaling laws in triadic resonances.

A linear stability analysis based on a mechanism of triadic resonance between Kelvin modes is currently under progress and will be the subject of a foregoing paper.

This study has been carried out under the contract CEA-CNRS N012171.
[1] W. V. R. Malkus. An experimental study of global instabilities due to tidal (elliptical) distortion of a rotating elastic cylinder. Geophys. Astrophys. Fluid Dynamics, 
48:123-134, 1989.

[2] A.D. McEwan. Inertial oscillations in a rotating fluid cylinder. J. Fluid Mech., 40(3):603-640, 1970.

[3] R. Manasseh. Breakdown regimes of inertia waves in a precessing cylinder. J. Fluid Mech., 243:261-296, 1992.

[4] R. Manasseh. Distortions of inertia waves in a rotating fluid cylinder forced near its fundamental mode resonance. J. Fluid Mech., 265:345-370, 1994

[5] R. Manasseh. Nonlinear behaviour of contained inertia waves. J. Fluid Mech., 315:151-173, 1996.

[6] J.J. Kobine. Azimutal flow assiociated with inertial wave resonance in a precessing cylinder. J. Fluid Mech., 319:387-406, 1996.

[7] A. Mahalov. The instability of rotating fluid columns subjected to a weak external coriolis force. Phys. Fluids A., 5:891-900, 1993.

[8] R.R. Kerswell. Secondary instabilities in rapidly rotating fluids: inertial wave breakdown. J. Fluid Mech., 382:283-
306, 1999 .

[9] J.J. Kobine. Inertial wave dynamics in a rotating and precessing cylinder. J. Fluid Mech., 303:233-252, 1995.

[10] P. Meunier, C. Eloy, R. Lagrange, and F. Nadal. A rotating fluid cylinder subject to weak precession. J. Fluid Mech., 599:405-440, 2008.

[11] P. Meunier and T. Leweke. Analysis and minimization of errors due to high gradients in particle image velocimetry. Exp. Fluids, 35(5):408-421, 2003.

[12] R.F. Gans. On the precession of a resonant cylinder. $J$. Fluid Mech., 41(4):865-872, 1970.

[13] C. Eloy, P. Le Gal, and S. Le Dizès. Elliptic and triangular instabilities in rotating cylinders. J. Fluid Mech., 476:357-388, 2003.

[14] R.R. Kerswell. Elliptical instability. Ann. Rev. Fluid Mech., 34:83-113, 2002. 